\title{
Using Body Scan Technology (Computer-Aided Anthropometry) to Measure Breast Volume ${ }^{1}$
}

\author{
Daisy VEITCH ${ }^{\star^{a}}$, Karen BURFORD ${ }^{b}$, Phil DENCH ${ }^{c}$, Nicola DEAN ${ }^{b}$, Philip GRIFFIN ${ }^{b}$ \\ a SHARP Dummies Pty Ltd, Belair (SA), Australia; \\ ${ }^{\mathrm{b}}$ Flinders Medical Centre, Bedford Park (SA), Australia; \\ cheadus (metamorphosis) Pty Ltd, Osborne Park (WA), Australia
}

\begin{abstract}
Assessment of breast volume is an important tool for preoperative planning in various breast surgeries and other applications, such as bra development. Accurate assessment can improve the consistency and quality of surgery outcomes. This study outlines a non-invasive method to measure breast volume using a whole body 3D laser surface anatomy scanner, the Cyberware WBX. It expands on a previous publication where this method was validated against patients undergoing mastectomy. It specifically outlines and expands the computer-aided anthropometric (CAA) method for extracting breast volumes in a non-invasive way from patients enrolled in a breast reduction study at Flinders Medical Centre, South Australia. This step-by-step description allows others to replicate this work and provides an additional tool to assist them in their own clinical practice and development of designs.
\end{abstract}

Keywords: anthropometry, breast volume, 3D scanning, computer-aided anthropometry, breast measurement

\section{Introduction}

Accurate determination of breast volume will help health professionals such as breast, plastic and reconstructive surgeons improve consistency in patient outcomes. They need to assess volume when determining asymmetry, for breast reduction and augmentation, and in breast reconstruction after mastectomy. Currently breast surgeons determine volume by eye $[7,10]$, which has the advantages of being fast, non-invasive and equipment-free, but also the disadvantage of being entirely dependent on the individual surgeon's skill and experience.

Physical methods to determine volume, such as casting (thermocasting plastic or plaster), or direct dipping of the breast in water to measure displacement, are invasive, time consuming and messy. Worse, these methods rely on false assumptions that limit their accuracy and validity.

Breast size or volume is difficult to determine. The volumetric 'gold standard' [21] for breast reconstruction purposes is the surgical removal of the entire breast (mastectomy), which is then weighed and placed in a plastic bag and immersed in water to determine the water displacement and hence the volume using Archimedes' principle [17]. This direct measurement technique is only available within and not prior to an operation. Therefore using this tool in preoperative planning is not possible.

Digital methods such as 3D laser scanning, magnetic resonance imaging, biostereometric analysis, computed tomography (CT), mammography, ultrasonography and others have also been used. This paper explores one digital method of image acquisition and computed-aided anthropometry (CAA), i.e., 3D laser scanning.

Measuring the human body requires at least two aspects to be satisfied. First, the measurement must be determined accurately, i.e., it would correspond to a real value and second, it must be valid, i.e., it measures the characteristic it is supposed to be measuring.

If accuracy is the extent to which a measured value corresponds to a real value [14] the question arises, what is the 'real value'? In anthropometry the real value is very difficult to determine. It is usually taken to be the value obtained by a skilled and experienced anthropometrist. In this case the accuracy of the volume will be dependent on where the edge of the breast is located and marked. The edge of the breast is sometimes referred to by surgeons as the breast base or breast footprint. Variation in this placement produces different volume measurements. Much of the breast tissue is fat

\footnotetext{
1 Acknowledgement to the AFESA team whose other members are Maciej Henneberg, Kalavani Zeitouneh, Phillipa Van Essen and others. We acknowledge and thank the Computerized Anthropometric Research and Design (CARD) laboratory, especially Dennis Burnsides and Kathleen Robinette who assisted in scanner selection and testing. Thanks also to Martin McAvoy for editorial assistance, Griselda Raisa Susanto for secretarial and research support and David Summerhayes for preparation of the images.

* daisy@sharpdummies.com.au
} 
and is non-differentiable from other body fat. As such it has no definitive edge recognizable by a change in tissue structure. Only the surgeon has at present the experience and the expertise to determine this crucial location.

A body cast of a large-breasted woman was developed by SHARP Dummies to provide a physical platform to test the proposed computer model. To measure volume we need a watertight 3D model. When the breast is cut from the torso a hole is created that needs to be filled and so a plane is necessary to close the gap in the geometry of the CAD model. This plane needs to follow the breast edge on the surface anatomy and realistically follow the shape of the torso as if a surgeon had cut off the real breast. This shape then becomes the geometry that creates the watertight 3D figure of the breast measurable as a volume. Therefore various shaped cut planes were tested.

Two surgeons were asked to physically indicate the edge of the breast and provide a realistic cut plane for the cast (see Figures 1 and 2). Almost immediately two things became apparent. First, the surgeons could not identify the exact margin of the breast in the absence of the soft tissue, and second, no flat cut plane could follow the body contour (see Figure 2).

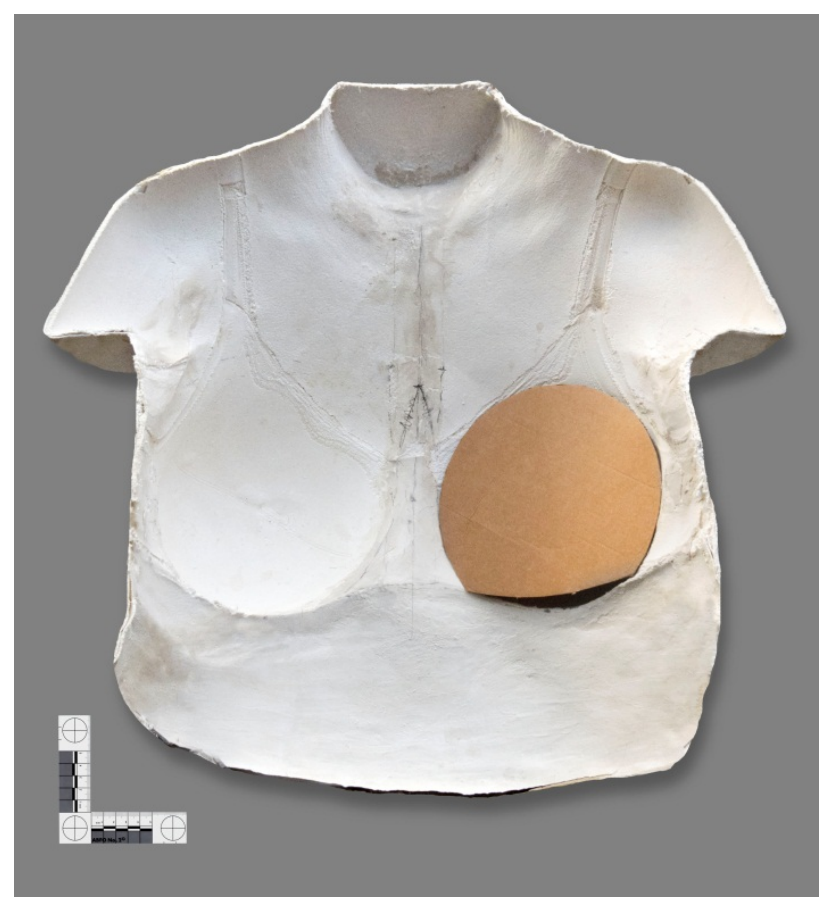

Fig. 1. Body cast viewed from the coronal plane used to problem solve the requirements for the volume extraction software. This method clearly revealed that a flat cut plane was insufficient for defining both the edge of the breast and the likely shape of the rib cage underneath.

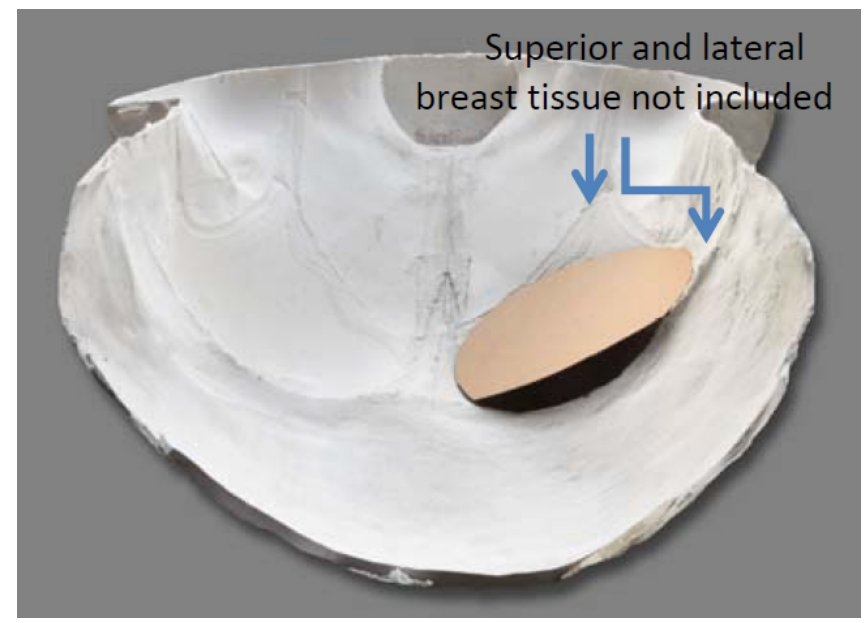

Fig. 2. Transverse view of cast (negative shape) looking towards the neck from the waist. A cardboard circle illustrates a flat cut plane. The gap shows the obvious inaccuracy of a flat cut plane as it deviates from the inframammary fold. 
The surgeons were unable to locate the breast margin because this is performed by palpation. Point locations cannot be determined by merely examining the surface. [16] The surgeon would have asked the patient to lift the breast until a crease appears at the superior edge, which is then marked. This is the top margin. Then the surgeon asks the patient to compress the breast laterally into the axilla region where again a crease appears which is manually marked. This becomes the lateral margin of the breast. Finally the inferior margin is taken to correspond with the inframammary fold. This way a differentiation between breast tissue and the surrounding adipose tissue is clearly delineated. There is no way this interactive method can be performed on a scan or body cast. These locations must be marked prior to image acquisition.

The cut plane is not flat because the inframammary fold follows the curved rib cage shape (see Figures 1 and 2) and the lateral aspect of the breast often curves into the axilla. An additional complication of the cut plane is caused by the convexity of the abdominal adiposity of patients with ptotic breasts.

Figures 3 and 4 show, when attempting to determine breast volume using water displacement of the in situ breast, the abdominal adiposity of the subject prevents the researcher from getting the flat plane of the water near the lateral (marked with white dot) and superior edges of the breast. The body cast (Figures 1 and 2) shows the same thing in a negative space as Figures 3 and 4 show in the positive. Abdominal adiposity is a substantial obstruction to the measurer reaching the lateral breast edge with the water's flat plane (Figure 4). Water displacement is an accurate technique, but in this case it is not measuring the breast in the correct place. Therefore the water volume is not the real breast volume.

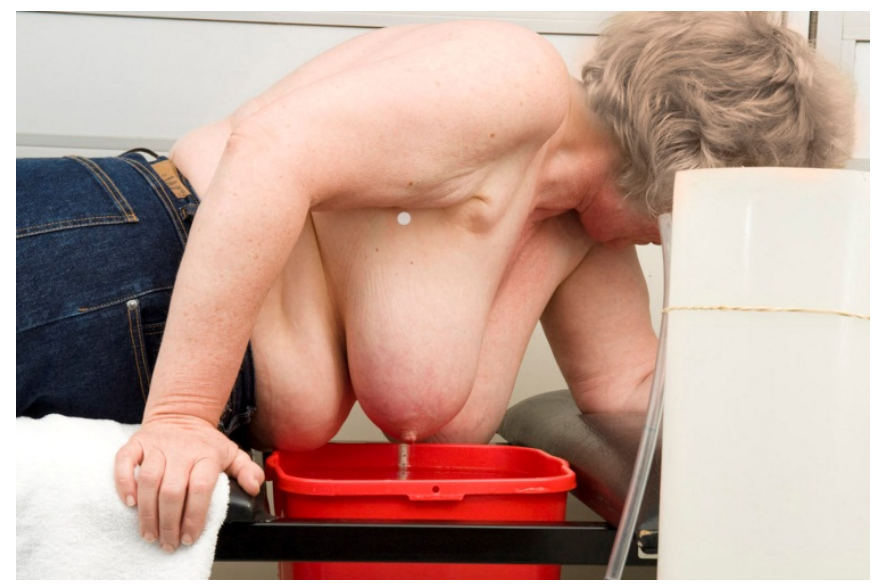

Fig. 3 shows the fat fold of the abdomen interfering with the measuring.

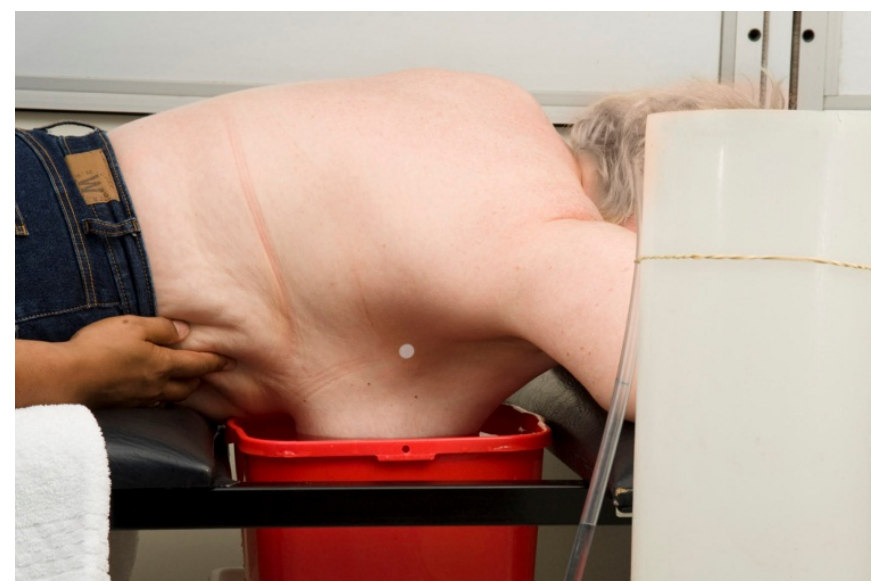

Fig. 4 shows the researcher attempting to hold the abdominal adiposity roll out of the bucket; however the superior and lateral (marked with white sticker) aspects of the breast edge are still nowhere near the surface of the water.

Therefore when measuring breast volume $[3-6,8,9,12,13,17,18,20]$ where the breast and body are intact, any method that relies on a flat cut plane is based on a conceptual fallacy: to try to follow the flat cut plane is not to follow the edge of the breast as determined by the surgeons. This immediately raises the question of validity. Does the method measure what it is supposed to i.e. breast volume? If the method can't follow the real edge of the breast, then no matter how accurate it is, the results will only be very approximate. 
So a reliable, accurate and valid tool needs to be developed that determines breast volume non-invasively. The method must build upon the surgeon's expertise in determining the edge of the breast, i.e., use palpation and markers, and the cut plane must be curved to follow both the surface breast boundary and the underlying anatomical structures of the rib cage - in the case of ptosis, the underlying abdominal shape.

\section{Methods}

This paper describes the method developed for the AFESA (Anthropometry for Elective Surgery Assessment) group for breast reduction research (not yet published), at Flinders Medical Centre, South Australia. Flinders Clinical Research Ethics Committee granted permission for the study (application \#269/08). This method has also been used in other studies at Flinders Medical Centre, including a mastectomy volume validation trial [21].

\subsection{Scanner selection}

A set of purchasing criteria was developed to select and test the whole body scanners using the following requirements:

- safety: eye and skin safe,

- high speed: avoid movement effects,

- data output type is point cloud: allows viewing and calibration of raw data,

- data output format in public domain (not proprietary): allow freedom to choose a third party software if required,

- resolution of $1 \mathrm{~mm}$ in all directions (or similar): closeness of 3D points to each other,

- precision: high accuracy of 3D points,

- ease of calibration: allows adjustment of raw data to fine tune accuracy,

- field of view at least $2 \mathrm{~m} \times 1.5 \mathrm{~m} \times 1.2 \mathrm{~m}$ or close: accommodates large subjects,

- good surface coverage: avoids holes in the field of view (minimal occlusion),

- good landmark recognition capability: ability to recognize flat markers, and

- warranty and support [15].

Scans were taken and tested at the Computerized Anthropometric Research and Design (CARD) laboratory in Ohio, USA prior to purchase.

As a result a Cyberware WBX [1] was chosen. The Cyberware laser scanner does not use x-rays or ionising radiation. It uses low-power red laser and infrared light to collect surface anatomy data only. It has no risks or harmful effects, as the laser is Class 1, which is considered safe enough to preclude a written warning on the device. The scanner has 4 scanheads that move vertically on pillars and scan the patient. An image of approximately 300,000 3D data points is acquired in 15 seconds and can immediately be checked for integrity on the attached computer. Time between scans can be as little as one minute.

Robinette and Daanen [16] have verified the precision of the Cyberware scanner as a measuring tool for CAA using preplaced landmarks with straight-line distances.

\subsection{Measurement extraction software}

A specification was written for the scanner software CAA extraction of breast volumes. Using the preplaced markers as a guide the software was required to select and use these points and create a curved plane defining the breast boundary.

As a result specialist software was written by headus using a mathematical averaging to create a contoured cut plane on the breast. This mimics the convex adiposity of the stomach and chest forming the underlying structure of the inframammary fold and breast base, and thus defines the volumetric boundary of the cut edge of the breast.

The mathematical position of each point defined on the edge of the breast cut plane is averaged in 3D to create one geometric centre or central locus. This locus is then joined to all other cut edge points to form a myriad of small triangular facets. These facets become the contoured plane face defining the volumetric boundary of the cut edge of the breast. The defined watertight 3D geometric shape is then divided into tetrahedrons, connecting each triangular face to the coordinate system origin, and the signed volumes of these tetrahedrons are summed to give total breast volume [11].

\subsection{Participants posture and garment selection}

Study participants were trialled in a number of scanning postures and scanning garments.

Candidates were scanned in two postures. The first was the CAESAR standardized Pose A: standing posture with the feet apart in footprints and arms adducted $30 \mathrm{cms}$ from the body [2]. The second pose 
is Pose C: arm position in the coronal plane with the humerus parallel to the floor, elbows at right angles and hands in the air. It differs from the CAESAR protocol in being used as a standing rather than a sitting pose. This allows a clear view of the underarm area, particularly in patients with ptotic triceps. A repeat of the first scan was made as a backup. Total measuring time is 15 minutes and post processing a further 30 minutes.

Upper garments were trialed on the women to try and standardize the scanning attire. CAESAR shorts and bras were trialled. The shorts, made of grey marl cotton elastomeric fibre, were ideal but the upper garments, designed to be worn over existing bras, showed significant occlusion of the breast area, in particular adding volume to the space between the breasts and changing the breast shape. Commercially purchased bras are limited in their range of sizes, in particular for large breasted women. A study of 939 women's bust and underbust measurements noted that 174 (nearly 20\%) were too large to fit into a regular bra. Almost all of the large size women had breasts C cup or larger. [19] No bras with a suitable fit for this population were found. The scanning of bare breasted participants resulted in no error from the scanning garment; but there was additional occlusion of the abdomen due to increased ptosis in some patients. However the 3D mathematical averaging managed to reproduce the shape of the underlying anatomical structures very well, see Figure 8 . Therefore in this study patients were scanned bare breasted.

\subsection{Prior marking and CAA}

The landmarking procedure to determine the breast perimeter was developed based on a standard technique surgeons employ for defining the edge of the breast on patients before breast surgery, using the palpation method already described in the introduction. The breast perimeter is marked with $10 \mathrm{~mm}$ diameter flat stickers prior to scanning (see Figure 5). These stickers define the precise location of the breast edge on the scanned data, thereby controlling the potential error of measuring in the wrong place.

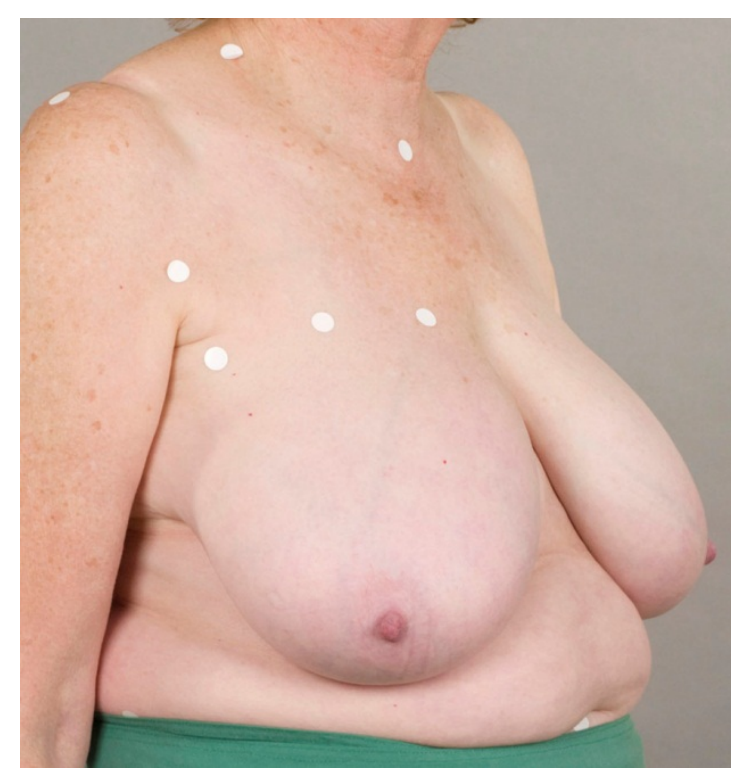

Fig. 5. Showing patient with the breast markers placed by palpation.

The patient is then photographed (hospital requirement), measured and scanned. Each scan is individually calibrated to the maximum alignment of the four output files. These four files are merged into one, landmarked electronically (see Figures 6 and 7), segmented (see Figure 8), and finally the volume is calculated. Left and right breast volumes are recorded. The scans and measurement files are saved and backed up on a server. 


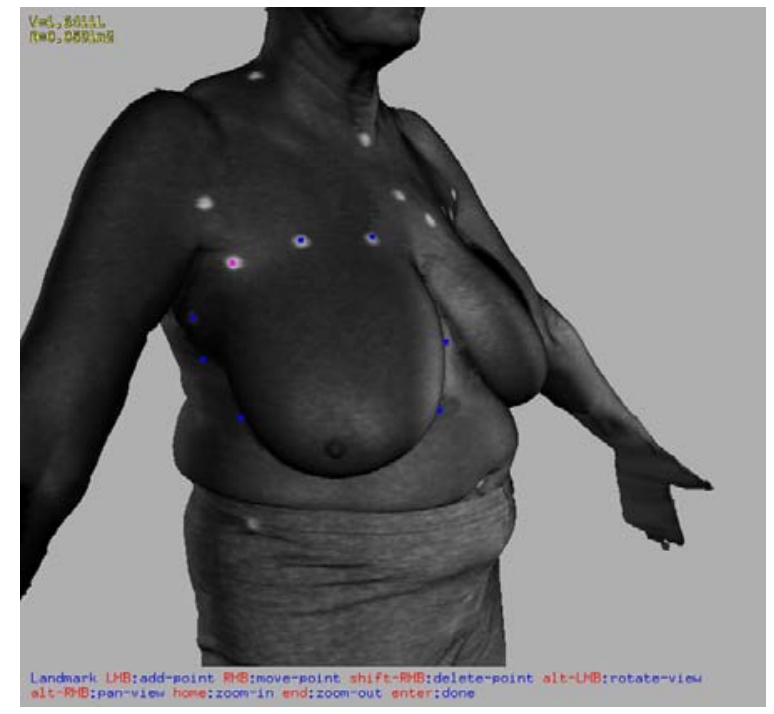

Fig. 6. Patient scan in luminance view highlighting the location of the markers and creating a separate measurement file matching the scan file.

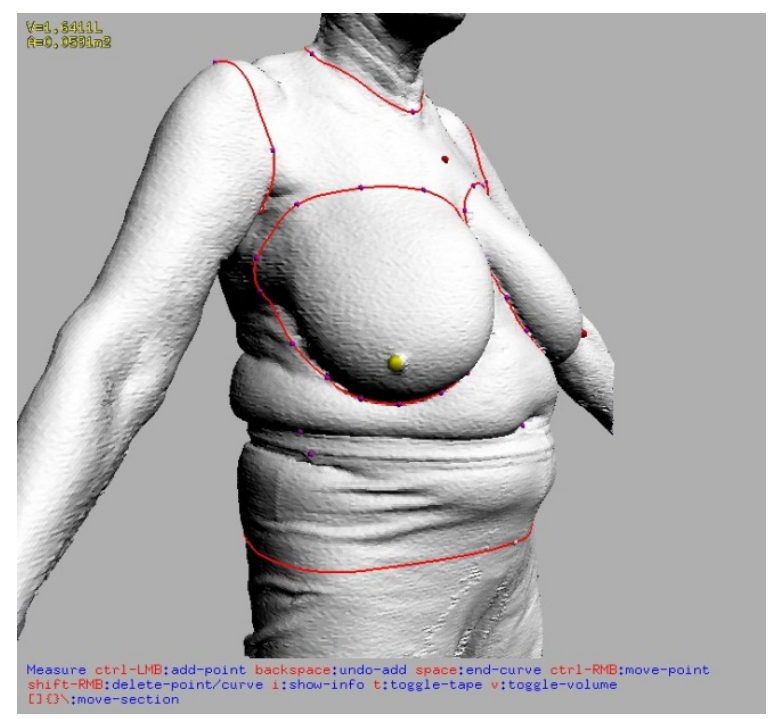

Fig. 7. Patient scan with breast boundaries marked following the preplaced markers.

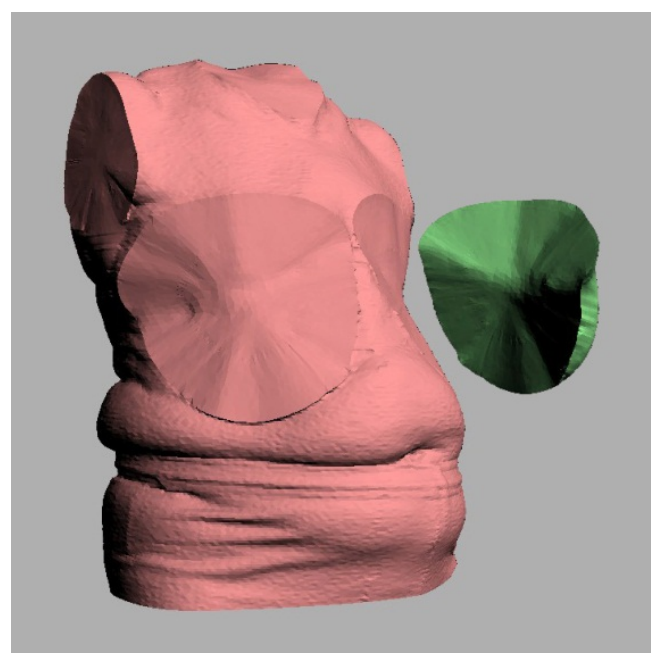

Fig. 8. It shows the cut plane created by the mathematical averaging that follows the chest wall and surface of the abdominal protuberance. The torso (pink) and the proximal surface view of the detached breast (green) illustrate different volume segments. 


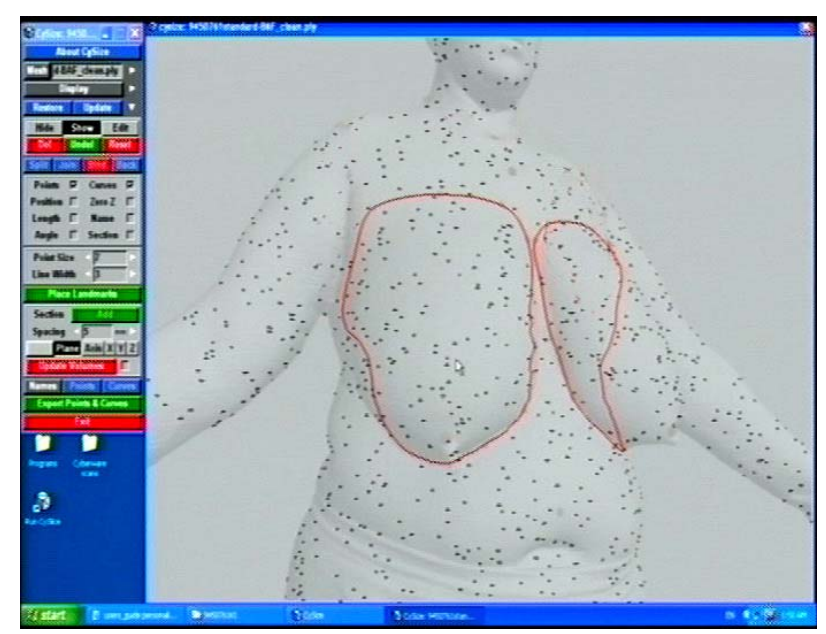

Fig. 9. It shows the red cut line of the plane segments. The dots (reduced in density for illustration purposes) indicate the data of the surface anatomy of the $3 D$ scan.

\section{Results}

This method has been validated using 30 patients who underwent 39 mastectomies. The mastectomy specimen was weighed and its volume calculated using direct water displacement, which is considered to be the gold standard (as distinct from water displacement of the in situ breast). The conclusions were that the volumes were very highly correlated at $r=0.095$ and the 3D scan volume of each individual breast was slightly higher than the mastectomy volume. This could have been due to skin being included in the scan volume calculation; the researchers proposed a formula to correct for this [21].

This method then is useful, accurate (as defined earlier) and valid because it measures what needs to be measured.

The technique is easy to use, reproducible and non-invasive. This method has been developed for and tested on some very difficult to measure patients with very large breasts and/or obesity. It is a population representative of and likely to present for breast reduction surgery. If the method can perform well for these patients it is likely to work for people closer to the idealized female shape, but this needs further testing.

The tool then is applicable to reconstruction, breast reduction and a range of other breast surgical procedures.

\section{Discussion}

In summary the usual methods of measuring breast volume in situ lack validity because they assume a flat cut plane that fails to follow the real edge of the breast (the breast base). In addition many of these methods are intrusive and messy. Direct water displacement measurement of mastectomy specimens, while being the volumetric gold standard of accuracy and validity, is only available for patients undergoing mastectomy and not for preoperative planning. The 3D laser scanning and CAA measurement extraction techniques described in this paper have managed to transfer the real breast edge on the real patient, with no loss of fidelity, into the virtual environment. This software creates the shaped cut plane that follows the underlying anatomical structure to produce the geometric shape required for volume calculation. These innovations address and resolve issues of validity and accuracy.

One limitation is that skin is included in the scan volume, whereas a mastectomy specimen does not include the portion of skin used to close the wound. As mentioned earlier, preliminary studies indicate this could be corrected by the formula described by Yip et al. [21].

3D laser scanning is very fast, only collecting surface information. But then occlusion may become a problem, especially in the population with a larger body mass index (BMI), not only occlusion due to breast ptosis, but also triceps ptosis where the loose skin occludes the side of the breast. The first has been addressed by the development of the complex cut plane shape following the abdominal curve, the second (in triceps ptosis) by the use of Scan 2 posture to raise the person's arms, thereby physically removing the arm flaps from occluding the lateral breast area. 
A scanner, such as the WBX, with a large accommodation volume, requires a room with higher than usual ceilings, more than $288 \mathrm{~cm}$ high. The photographic studio based in Flinders Medical Centre, Department of Media and Illustration, met this requirement.

The method outlined here only addresses the measurement of volume, one indication of size, but there are applications for assessing other anthropometric characteristics. Symmetry involves size, location and shape assessment, and these when captured allows significant comparisons.

These technologies are being used in other studies to measure location, which could include inframammary height or nipple height to ground, and/or shape variations such as those of breast base area, and 3D surface topology. 3D digital technologies, like the one used in this study, permanently store scans that can be re-interrogated later for future study, or to check previous work using more advanced software as it is developed. This is a significant advance over 1D anthropometric measuring where shape and location data are very difficult to acquire, and once the subject leaves it is almost impossible to acquire additional data.

This study validates:

1. the physical method of palpation to find the breast edge or base,

2. premarking of the person prior to scanning,

3. image acquisition by Cyberware WBX and CyScan, and

4. CAA (measurement extraction) using Cy-Slice software for the determination of breast volume.

It is applicable as a tool in, for example, preoperative breast surgery planning. This method is particularly valuable in being developed for and tested on participants typically difficult to measure, i.e. breast reduction patients.

Independent validation is still necessary for other scanners and software.

\section{Acknowledgment}

A version of this paper will be published in a special issue of the Journal Work: A Journal of Prevention, Assessment \& Rehabilitation (IOS Press), 2012.

\section{References}

[1] Anonymous, Cyberware, Whole Body Color 3D Scanner (Model WBX) Specifications, 1999, http://www.cyberware.com/products/scanners/wbxSpecs.html

[2] S. Blackwell, K.M. Robinette, H.A.M. Daanen, M. Boehmer, S. Fleming, S. Kelly, T. Brill, D. Hoeferlin, and D. Burnsides, Technical report ARFL-HE-WP-TR-2002-0173, United States Air Force Research Laboratory, Civilian American and European surface anthropometry resource (CAESAR), Final Report 2, Warrendale, PA.

[3] F.G. Bouman, Volumetric measurement of the human breast and breast tissue before and during mammaplasty, British Journal of Plastic Surgery 23 (1970), 263-264.

[4] N. Bulstrode, E. Bellamy, and S. Shrotria, Breast volume assessment: comparing five different techniques, The Breast 10 (2001), 17-123.

[5] B.N. Campaigne, V.L. Katch, P. Freedson, S. Sady, and F.I. Katch, Measurement of breast volume in females: description of a reliable method, Annals of Human Biology 6 (1979), 363-367.

[6] A. Edsander-Nord, M. Wickman, and G. Jurell, Measurement of breast volume with thermoplastic casts, Scandinavian journal of plastic and reconstructive surgery and hand surgery 30 (1996), 129-132.

[7] D.A. Hudson, Factors determining shape and symmetry in immediate breast reconstruction, Annals of Plastic Surgery 52 (2004), 15-21.

[8] $\mathrm{H}$. Ingleby, Changes in breast volume in a group of normal young women, Bulletin of the International Association of Medical Museums 29 (1949), 87-92

[9] V.L. Katch, B. Campaigne, P. Freedson, S. Sady, F.I. Katch, and A.R. Behnke, Contribution of breast volume and weight to body fat distribution in females. American journal of physical anthropology 53 (1980), 93-100.

[10] L. Kovacs, A. Zimmerman, N.A. Papadopoulos et al, Re. Factors determining shape and symmetry in immediate breast reconstruction, Annals of Plastic Surgery 53 (2004), 192-194.

[11] B. Mirtich, Fast and Accurate Computation of Polyhedral Mass Propoerties, Journal of Graphics Tools, Volume 1, Number 1, 1996.

[12] A.M. Nassar and R.E. Smith, Menstrual variations in thermal properties of the human breast. Journal of applied physiology 39 (1975), 806-811. 
[13] W.E. Palin, J.A. von Fraunhofer, and D.J. Smith, Measurement of breast volume: comparison of techniques. Plastic and reconstructive surgery 77 (1986),253-255.

[14] D. Pederson and C. Gore, Anthropometry Measurement Error, Chapter 3 in Anthropometry, ed. K. Norton and T. Olds, 1996, p.79.

[15] K.M. Robinette, Body Scanner Standards Overview oral presentation given at ASTM, D13 meeting, Washington DC, 2005

[16] K. M. Robinette and H. A. M. Daanen, Precision of the CAESAR scan-extracted measurements, Applied Ergonomics 37 (2006), 259-265.

[17] R.C. Schultz, R.F. Dolezal, J. Nolan, Further applications of Archimedes' principle in the correction of asymmetrical breasts. Annals of Plastic Surgery 16 (1986), 98-101.

[18] D.J. Smith, W.E. Palin, V.L. Katch, and J. E. Bennett, Breast volume and anthropomorphic measurements: normal values. Plastic and reconstructive surgery 781986;78:331-335.

[19] D. Veitch, R. Dawson, H. Owen, and C. Leigh, The Development of a Lifelike Breast Cancer Patient Simulator using Anthropometric Data 2011) Ergonomics Australia Journal - HFESA 2011 Conference Edition, 2011 11:45. http://www.ergonomics.org.au/resource_library/journal.aspx

[20] J.H. Wilmore, A.E. Atwater, B.D. Maxwell, D.L. Wilmore, S.H. Constable and M.J. Blonde, Alterations in breast morphology consequent to a 21-day bust developer program, Medicine and science in sports and exercise 17 (1985), 106-112.

[21] J.M. Yip, N. Mouratova, R.M. Jeffery, D.E. Veitch, R.J. Woodman and N.R. Dean, Accurate Assessment of Breast Volume: A Study Comparing the Volumetric Gold Standard (Direct Water Displacement Measurement of Mastectomy Specimen) With a 3D Laser Scanning Technique, Annals of Plastic Surgery May 16 2011, EPub Ahead of Print. 